\title{
c-Jun suppresses the expression of WNT inhibitory factor 1 through transcriptional regulation and interaction with DNA methyltransferase 1 in gallbladder cancer
}

\author{
BIN LIN ${ }^{1,2,4}$, HAIJIE HONG ${ }^{1,2}$, XIAOJIE JIANG ${ }^{1,2}$, CHENGZONG LI $^{1,2}$, SIYUAN ZHU $^{1,2}$, \\ NANHONG TANG ${ }^{1}$, XIAOQIAN WANG ${ }^{1}$, FEIFEI SHE ${ }^{2,3}$ and YANLING CHEN ${ }^{1,2}$
}

${ }^{1}$ Department of Hepatobiliary Surgery and Fujian Institute of Hepatobiliary Surgery, Fujian Medical University
Union Hospital, Fuzhou, Fujian 350001; ${ }^{2}$ Key Laboratory of Ministry of Education for Gastrointestinal Cancer;
${ }^{3}$ Fujian Key Laboratory of Tumor Microbiology, Fujian Medical University, Fuzhou, Fujian 350108, P.R. China

Received November 21, 2016; Accepted July 4, 2017

DOI: $10.3892 / \mathrm{mmr} .2018 .8890$

\begin{abstract}
WNT inhibitory factor $1(W I F-1)$ is involved in the tumorigenicity and progression of several types of tumor, which has been attributed to aberrant hypermethylation of its promoter. However, the role of WIF-1 in the pathogenesis of gallbladder cancer (GBC) remains to be fully elucidated, and the data available are insufficient to identify the upstream molecular mechanisms involved. In the present study, the methylation status of the WIF-1 promoter was investigated using methylation-specific polymerase chain reaction (PCR) and bisulfate sequencing PCR in GBC cells. Immunohistochemistry, reverse transcription-quantitative PCR and western blotting were used to analyze the expression of WIF-1 and c-Jun. In addition, a co-immunoprecipitation assay was designed to determine the DNA methyltransferase that was implicated in $W I F-1$ methylation. The results revealed that the expression of WIF-1 was low in GBC, and that this was caused by aberrant DNA hypermethylation. However, there were no significant correlations between the expression of WIF-1 and certain key clinicopathological characteristics of GCB. Subsequently, a negative correlation
\end{abstract}

Correspondence to: Dr Yanling Chen, Department of Hepatobiliary Surgery and Fujian Institute of Hepatobiliary Surgery, Fujian Medical University Union Hospital, 29 Xin-Quan Road, Fuzhou, Fujian 350001, P.R. China

E-mail: drchenyl@yeah.net

Dr Feifei She, Key Laboratory of Ministry of Education for Gastrointestinal Cancer, Fujian Medical University, 1 Xue-Yuan Road, Fuzhou, Fujian 350108, P.R. China

E-mail: shefeifei@yeah.net

Present address: ${ }^{4}$ Fujian Center for Food and Drug Certification and Evaluation, Fuzhou, Fujian 350001, P.R. China

Key words: gallbladder cancer, WNT inhibitory factor 1, c-Jun, methylation, DNA methyltransferase 1 was found between the protein expression of c-Jun and WIF-1 in 50 GBC specimens using immunohistochemistry. The demethylation and re-expression of $W I F-1$ was observed when the expression of $c$-Jun was silenced. Finally, it was found that the knockdown of $c$-Jun downregulated the expression of DNA methyltransferase 1 (DNMT1) and that c-Jun interacted with DNMT1. Taken together, the present study suggested that $c$-Jun suppressed the expression of WIF-1 through transcriptional regulation and interaction with DNMT1 in GBC. These findings provide an alternative pathogenesis of $\mathrm{GBC}$, which may be promising as a novel reference for early diagnosis or future treatment.

\section{Introduction}

Gallbladder cancer (GBC) is the most common and aggressive type of malignant tumor of the biliary tract in humans (1). The lack of markers for the timely prediction and diagnosis of $\mathrm{GBC}$, and its frequently silent and rapid progression confer a poor prognosis. Statistics show that, due to failure in promptly diagnosing GBC, $<10 \%$ of patients are suitable to undergo curative resection (2). Furthermore, the overall mean survival rate is only 6 months in the majority of patients, even following surgery, and the five-year survival rate is only $5 \%$ (3). To date, the elucidation of several molecular mechanisms has revealed the roles of genetic and epigenetic changes involved in the tumorigenicity and progression of human cancer. However, these factors in GBC remain to be fully elucidated.

The aberrant methylation of DNA, the most well known type of epigenetic modification, occurs at $\mathrm{CpG}$ islands in promoter regions and generally leads to methylation-based downregulation or silencing of gene expression. The downregulation of tumor suppressors, including P53 (4), phosphatase and tensin homolog (5), cyclin D2 (6) and Wnt inhibitory factor-1 (WIF-1) (7), caused by aberrant promoter methylation is integral in the occurrence and development of several types of cancer. Modifications in methylation are mediated by DNA methyltransferases (DNMTs), including DNMT1, DNMT3a, and DNMT3b. DNMT1 maintains its methyltransferase activity to reestablish methylation patterns 
throughout the DNA synthesis process, whereas DNMT3a and DNMT3b act as de novo methyltransferases (8).

$W I F-1$, an antagonist of the $\mathrm{Wnt} / \beta$-catenin pathway has been demonstrated to be an important tumor suppressor (7). As a secreted frizzled-related protein, WIF-1 can directly bind to Wnt molecules at the cell surface, thereby preventing Wnt from binding to receptors (9). It has been demonstrated that the expression of $W I F-1$ is decreased in several types of cancer, including breast cancer (10), lung cancer (11) and cervical cancer (12). The low expression of WIF-1 leads to abnormal activation of the Wnt pathway, resulting in the dysregulation of cell proliferation and differentiation, and inducing carcinogenesis in humans. There is also increasing evidence supporting the hypothesis that low expression levels of WIF-I can largely be attributed to aberrant hypermethylation of its promoter region (10-12). In our previous study, it was demonstrated that WIF- 1 was downregulated in GBC cell lines and tissues, which led to aberrant activation of the Wnt pathway, altering the processes of proliferation, invasion, metastasis and apoptosis; WIF- 1 was involved in the tumorigenicity and progression of GBC through these effects (13). However, the upstream molecular mechanism regulating WIF-1 remains to be fully elucidated, particularly in GBC.

$c$-Jun, the first oncogenic transcription factor to be identified, is the cellular homolog of the viral oncoprotein v-Jun, which is transcriptionally activated at Ser63 and Ser73 by Janus kinase (14). Early experiments revealed that the cooperation between $c$-Jun and oncogenic RAS is involved in tumor initiation and increased invasiveness in humans $(15,16)$. In addition, $c$-Jun has been shown to lead to loss of function of the tumor suppressor p53 $(17,18)$. $c$-Jun is widely expressed in different human tumors, is involved in numerous cell signaling pathways, and contributes to the pathogenesis, invasion and metastasis of cancer through diverse mechanisms $(19,20)$.

The present study provided novel evidence demonstrating that the low expression of WIF-1 in GBC cells was caused by aberrant hypermethylation of its promoter. The results revealed a negative correlation between the protein levels of c-Jun and WIF-1 in 50 GBC specimens. It was subsequently determined that the knockdown of $c$-Jun using RNA interference restored the expression of WIF-1. Based on these results and previous findings, it was hypothesized that $c$-Jun affects the process of methylation, particularly that performed by major methyltransferases, which leads to hypermethylation and downregulation of the WIF-1 gene in GBC.

\section{Materials and methods}

Specimens. A total of 50 GBC sample tissues and 20 cholecystitis tissues were obtained from patients at the Department of Surgery and Pathology of Fujian Medical University Union Hospital (Fujian, China) between 2006 and 2013. None of the GBC patients had received any preoperative chemotherapy or radiotherapy. The tissue samples were fixed in $10 \%$ buffered formalin and embedded in paraffin wax. Written consent was obtained from each patient to perform experiments on the resected specimens, and the study was approved by the ethnical committee of the Medical Faculty of Fujian Medical University in accordance with the 1975 Declaration of Helsinki.
Immunohistochemistry and evaluation. Serial $4 \mu \mathrm{m}$ sections were obtained from the formalin-fixed and paraffin-embedded tissues. Following deparaffinization in turpentine and rehydration in an alcohol gradient, the tissue sections were incubated in $3 \%$ hydrogen peroxide for $10 \mathrm{~min}$ at room temperature $\left(\sim 25^{\circ} \mathrm{C}\right)$ to prevent the activity of endogenous peroxidases. The tissue sections were subjected to antigen retrieval through boiling in citrate buffer ( $\mathrm{pH} 6.0$ ) for $10 \mathrm{~min}$ in a microwave, following which they were cooled at room temperature for 45 min and washed with phosphate-buffered saline (PBS). Non-specific antigens in the sections were blocked via incubation for $20 \mathrm{~min}$ in 5\% normal goat serum (Beyotime Institute of Biotechnology, Shanghai, China) diluted in PBS. The tissue sections were subsequently incubated in a 1:100 dilution of a rabbit polyclonal anti-human c-Jun antibody (cat. no. ab31419; Abcam, Cambridge, UK) or a 1:100 dilution of a rabbit polyclonal anti-human WIF-1 antibody (cat. no. ab186845; Abcam) overnight in humidified boxes at $4^{\circ} \mathrm{C}$. Following being washed in PBS, the sections were incubated according to the instructions provided with the UltraSensitive S-P kit (Maixin-Bio, Fuzhou, China). Prior to being dehydrated and mounted, the sections were stained with 3,3'-diaminobenzidine (DAB) for 3-5 min and counterstained using hematoxylin for $15 \mathrm{~min}$. PBS was substituted for the primary antibody as a negative control. Cells showing deposition of buff-colored granules in the cytoplasm and nucleus were scored as c-Jun- or WIF-1-positive. The expression levels of c-Jun or WIF-1 were semiquantitatively analyzed using the mean optical density (MOD), defined as the integral optical density/positive area, calculated using Image-Pro Plus 6.0 software (Media Cybernetics, Inc., Rockville, MD, USA).

Cell culture and treatment. The human GBC cell lines, NOZ (Health Science Research Resources Bank, Osaka, Japan), GBC-SD (Shanghai Institutes for Biological Sciences, Shanghai, China) and SGC-996 (Tumor Cytology Research Unit, Medical College, Tongji University, Shanghai, China) were cultured in Dulbecco's modified Eagle's medium (DMEM; Gibco; Thermo Fisher Scientific, Inc., Waltham, MA, USA) containing $10 \%$ fetal bovine serum (FBS; HyClone, GE Healthcare Life Sciences, Logan, UT, USA) under $5 \% \mathrm{CO}_{2} / 95 \%$ air, in a humidified $37^{\circ} \mathrm{C}$ incubator.

The GBC cell lines were divided into 6 -well plates at a density of $2 \times 10^{5}$ cells/well ( $\sim 30 \%$ confluence) $12 \mathrm{~h}$ prior to treatment. The cells were treated with 5-aza-2'-deoxycytidine (DAC; Sigma-Aldrich; Merck Millipore, Darmstadt, Germany) dissolved in dimethyl sulfoxide at a concentration of $5.0 \mu \mathrm{M}$, which was replaced every $24 \mathrm{~h}$ for a total treatment duration of $72 \mathrm{~h}$.

Cell transfection. Appropriate small interfering (si)RNA target sequences were determined based on the human $c$-Jun sequence (GenBank accession no. NM_002228.3). DNA template oligonucleotides corresponding to three siRNA sequences were designed according to the guidelines for siRNA design: P-1 sense, 5'-GGACCUUAUGGCUACAGUATT-3' and antisense, 5'-UACUGUAGCCAUAAGGUCCTT-3'; P-2 sense, 5'-ACG CAAACCUCAGCAACUUTT-3' and antisense, 5'-AAGUUG CUGAGGUUUGCGUTT-3'; and P-3 sense, 5'-GGAACAGGU GGCACAGCUUTT-3' and antisense, 5'-AAGCUGUGCCAC 
Table I. Primers used for polymerase chain reaction and sequence analyses.

\begin{tabular}{lll}
\hline Gene & \multicolumn{1}{c}{ Forward primer } & \multicolumn{1}{c}{ Reverse primer } \\
\hline WIF-1 & 5'-CCAGGACTAGAGGGAGAGCA-3' & 5'-TCGCAGACAGGCTTTGAACA-3' \\
$c$-Jun & 5'-AGGAAGCTGGAGAGAATCGC-3' & 5'-GTTAGCATGAGTTGGCACCC-3' \\
DNMT 1 & 5'-AGAAGTGAAGCCCGTAGAGTG-3' & 5'-ATGAGATGTGATGGTGGTTTGG-3' \\
DNMT3b & 5'-TGTAACGAAGTGAAGGAGGAGAA-3' & 5'-CATCTTGCCGAGGGAGTCT-3' \\
GAPDH & 5'-AGAGGAGTGTGAAGCAAGGA-3' & 5'-TGAGAAATGAGGGTAGCAGACT-3' \\
USP $(W I F-1)$ & 5'-AGGGCTGCTTTTAACTCTGGT-3' & 5'-TCTCGCTCCTGGAAGATGGTG-3' \\
MSP $(W I F-1)$ & 5'-GAATTTTATTGGTTGAAAGGGAGAT-3' & 5'-AAAAATAAAAAAAACAAACAACACT-3' \\
BSP $(W I F-1)$ & 5'-AATTTTATTGGTTGAAAGGGAGAC-3' & 5'-AAAAATAAAAAAAACAAACAACGCT-3' \\
& 5'-GGAATTTTTAAATGTTGGGTGT-3' & 5'-AAATAATAACTCCTATTCCTCCTCC-3'
\end{tabular}

WIF-1, WNT inhibitory factor 1; DNMT, DNA methyltransferase; GAPDH, glyceraldehyde-3-phosphate dehydrogenase; USP, unmethylation-specific primer; MSP, methylation-specific primer; BSP, bisulfate sequencing polymerase chain reaction.

CUGUUCCTT-3', which were constructed by GenePharma Co., Ltd. (Shanghai, China). A non-targeting siRNA was used as a negative control (NC-siRNA).

Transfection was performed with Lipofectamine 2000 (Invitrogen; Thermo Fisher Scientific, Inc.) according to the manufacturer's protocol, when the NOZ cells were at $\sim 90 \%$ confluence. The transfection efficiency was evaluated by counting the percentage of enhanced green fluorescent protein-positive NOZ cells using a fluorescence microscope. The cells were harvested $48 \mathrm{~h}$ following transfection to extract mRNA for reverse transcription-quantitative polymerase chain reaction (RT-qPCR) analysis and at $72 \mathrm{~h}$ to extract protein for western blot analysis.

RNA extraction and RT-qPCR analysis. Total RNA was extracted from the GBC cells with TRIzol reagent (Invitrogen; Thermo Fisher Scientific, Inc.) and then quantified using a spectrophotometer. cDNA was synthesized with the Revert Aid First-Strand cDNA Synthesis kit (Thermo Fisher Scientific, Inc.) using $2 \mu \mathrm{g}$ of RNA according to the manufacturer's protocol. Glyceraldehyde-3-phosphate dehydrogenase $(G A P D H)$ was used as an internal control. The primers for WIF-1, c-Jun, DNMT1, DNMT3a, DNMT3b and GAPDH were designed and synthesized according to standard primer design principles (Table I). PCR reactions were performed using FastStart Universal SYBR-Green Master Mix (Roche Diagnostics, Basel, Switzerland) on a 7500 Fast Real-Time PCR system (Applied Biosystems; Thermo Fisher Scientific, Inc.). The following PCR conditions were used: Initial denaturation at $95^{\circ} \mathrm{C}$ for $3 \mathrm{~min}$, followed by 40 cycles at $95^{\circ} \mathrm{C}$ for $15 \mathrm{sec}$ and at $60^{\circ} \mathrm{C}$ for $30 \mathrm{sec}$. Each sample was analyzed in triplicate. The relative expression levels of target genes were calculated based on normalization to the endogenous mRNA expression of $G A P D H$, prior to comparative analysis using the $2^{-\Delta \Delta \mathrm{Cq}}$ method (21).

Protein extraction and western blot analysis. For protein extraction, the cells were lysed in RIPA lysis buffer containing a protease inhibitor mixture. The protein concentration was then measured using a bicinchoninic acid assay (Beyotime Institute of Biotechnology) according to the manufacturer's protocol. A total of $20 \mu \mathrm{g}$ of protein was subjected to $10 \%$ sodium dodecyl sulphate-polyacrylamide gel electrophoresis (SDS-PAGE) and then transferred onto a polyvinylidene fluoride (PVDF) membrane (GE Healthcare Life Sciences, Chalfont, UK). Following blocking with 5\% nonfat milk for $3 \mathrm{~h}$, the PVDF membranes were separately incubated with primary antibodies against c-Jun (1:1,000 dilution; cat. no. ab31419; Abcam), WIF-1 (1:1,000 dilution; cat. no. ab186845; Abcam), DNMT1 (1:1,000 dilution; cat. no. 5119; Cell Signaling Technology, Inc., Danvers, MA, USA), DNMT3a (1:1,000 dilution; cat. no. 3598; Cell Signaling Technology, Inc.), DNMT3b (1:1,000 dilution; cat. no. 67259; Cell Signaling Technology, Inc.) and $\beta$-actin $(1: 1,000$ dilution; cat. no. sc-47778; Santa Cruz Biotechnology, Santa Cruz, CA, USA) overnight at $4^{\circ} \mathrm{C}$. Following several washes with Tris-buffered saline with Tween (TBST), the appropriate secondary antibody conjugated to horseradish peroxidase (1:2,000 dilution; cat. no. ZB-2301 or ZB-2305; ZSGB-BIO, Beijing, China) was added to the membranes, followed by incubation for $1 \mathrm{~h}$ at room temperature. The quantities of each protein were visualized using ECL Advance reagent following use of a chemiluminescence western blot immunodetection kit (Invitrogen; Thermo Fisher Scientific, Inc.).

DNA extraction, methylation-specific PCR and bisulfate sequencing PCR (BSP). Genomic DNA was isolated from the cells and tissues using the TIANamp Genomic DNA kit (Tiangen Biotech Co., Ltd.) according to the manufacturer's protocol. Subsequently, $2 \mu \mathrm{g}$ of genomic DNA was converted with sodium bisulfite and subsequently cleaned using a commercial kit (EpiTect Bisulfite kit; Qiagen GmbH, Hilden, Germany).

EMBOSS CpGplot software (http://www.ebi.ac.uk/Tools/ seqstats/emboss_cpgplot/) (22) was use to predict a CpG island spanning nucleotides -562 to -308 following depositing the 2,000 bp prior to the transcriptional start site of the WIF-1 gene promoter sequence (NC_000012), in which $19 \mathrm{CpG}$ dinucleotides were present (Fig. 1A). The primers used to amplify bisulfite-treated genomic DNA (nucleotides -557 to -302) for BSP were designed using MethPrimer (http://www. urogene.org/cgi-bin/methprimer/methprimer.cgi) (23) and are listed in Table I. The amplified products were extracted 


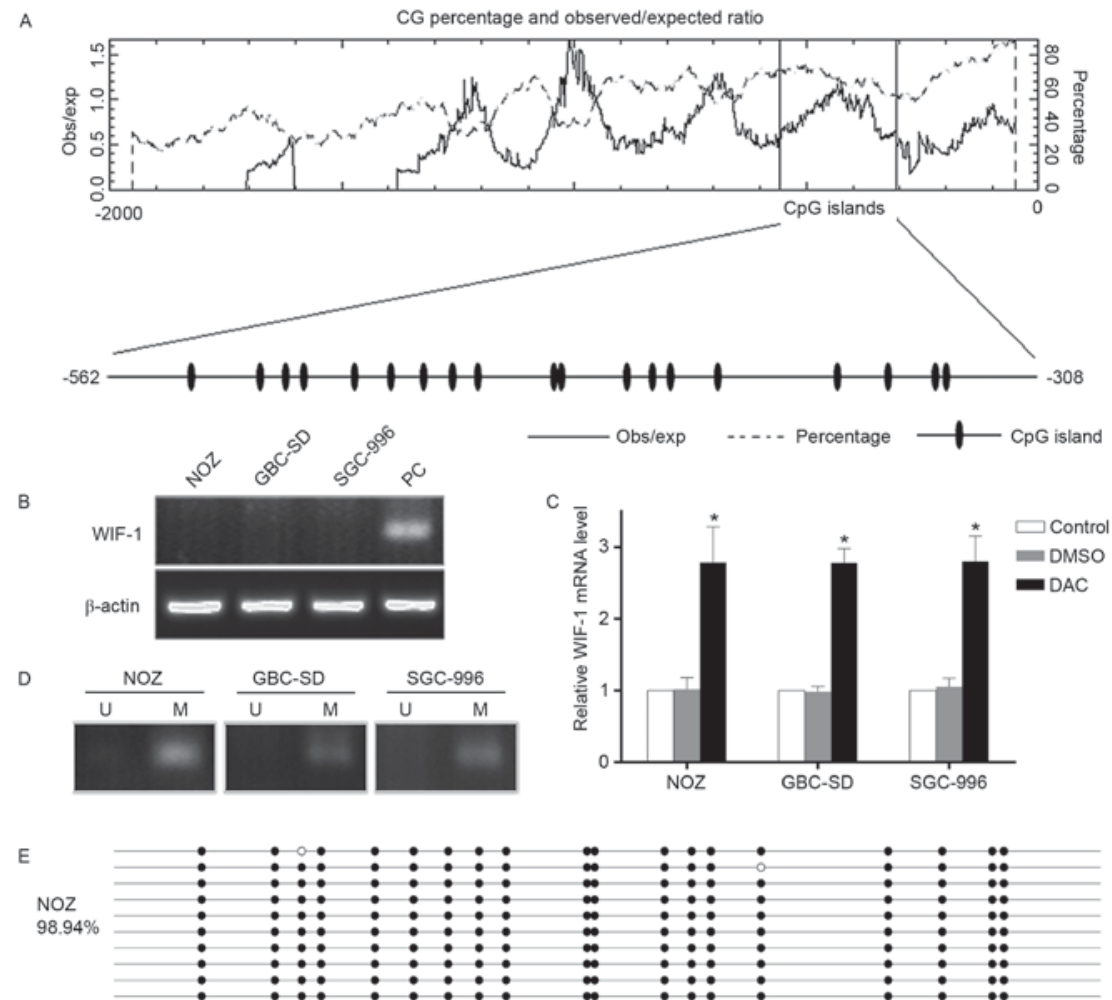

Figure 1. Loss of the expression of WIF-1 and aberrant hypermethylation of its promoter region in GBC cells. (A) A putative CpG island containing 19 CpG dinucleotides relative to the transcriptional start site meeting the following criteria: Percent $\mathrm{C}+$ percent $\mathrm{G}>50 \%$; obs/exp ratio $>0.60$; length $>200$ bp. (B) Relative mRNA level of WIF-1 in GBC cell lines. $\beta$-actin served as a loading control. (C) mRNA expression of WIF-1 following DAC treatment in GBC cell lines. "P $<0.05$, vs. control group. (D) methylation-specific PCR assay of GBC cells. M bands are visible; U bands are not visible. (E) Bisulfite genomic sequencing of the WIF-1 promoter region in NOZ cells. Each circle corresponds to one CpG position; filled (black) circles correspond to methylated Cs; unfilled (white) circles correspond to unmethylated Cs. The percentage of methylated Cs was $98.94 \%$. WIF-1, WNT inhibitory factor 1; GBC, gallbladder cancer; M, methylation-specific primer; U, unmethylation-specific primer; PC, positive control normal human lung tissue; obs, observed; exp, expected; DMSO, dimethyl sulfoxide; DAC, 5-aza-2'-deoxycytidine.

from a $1.5 \%$ agarose gel stained with ethidium bromide, using a gel extraction kit (E.Z.N.A.TM Gel extraction kit; Omega Bio-Tek, Inc., Norcross, GA, USA) and then purified for subcloning into the pMD18-T vector (Takara Bio, Inc., Otsu, Japan). Subsequently, 10 clones were randomly selected from each sample to determine the methylation status of the $\mathrm{CpG}$ islands of the WIF-1 promoter. The status of DNA methylation was determined using BiQ Analyzer software version 2.00 (http://biq-analyzer.bioinf.mpi-inf.mpg.de) (24).

For the methylation-specific PCR assay, bisulfite-treated genomic DNA was amplified using either an unmethylation-specific primer (USP) or a methylation-specific primer (MSP), as listed in Table I, targeting the WIF-1 promoter region sequence from $-331 /-330$ to $-164 /-164$, including five $\mathrm{CpG}$ islands. In this assay, results were defined as positive results when MSP bands were visible, with negative results defined as those with visible USP bands with or without MSP bands.

Co-immunoprecipitation assay. Soluble proteins were precleared with $1.0 \mu \mathrm{g}$ of normal rabbit $\operatorname{IgG}$ and $20 \mu \mathrm{l}$ of the resuspended volume of protein A/G plus-agarose (Santa Cruz Biotechnology, Inc.). Subsequently, $1 \mathrm{mg}$ of total cellular protein was mixed with $3 \mu \mathrm{g}$ of primary antibodies (c-Jun or DNMT1), following which $20 \mu \mathrm{l}$ of the resuspended volume of protein $\mathrm{A} / \mathrm{G}$ plus-agarose was added, and the samples were incubated at $4^{\circ} \mathrm{C}$ on a rotating device overnight. The immunoprecipitated complexes were washed with lysis buffer and then analyzed via 10\% SDS-PAGE and western blot analysis, using the respective specific antibodies.

Statistical analysis. The statistical analyses were performed using SPSS software version 16.0 (SPSS, Inc., Chicago, IL, USA) or GraphPad Prism software version 6.0 (GraphPad Software, Inc., La Jolla, CA, USA). Fisher exact test assessed the associations between the protein expression of WIF-1 and clinical pathological parameters. Pearson's coefficient was used for the calculation of correlations of the MOD values of WIF-1 and c-Jun, which represented the protein levels in GBC tissues. The measurement data are expressed as the mean \pm standard deviation from at least three independent experiments and were analyzed using independent samples t-tests. Two-sided $\mathrm{P}<0.05$ (two-sided) was considered to indicate a statistically significant difference.

\section{Results}

Loss of the expression of WIF-1 and aberrant hypermethylation of its promoter region in GBC cells. The results of the RT-qPCR analysis revealed the complete loss of WIF-1 in the GBC (NOZ, GBC-SD and SGC-996) cell lines analyzed (Fig. 1B). Treatment with the demethylating agent DAC effectively restored the mRNA levels of WIF-1 (Fig. 1C), suggesting that genome 
Table II. Association between clinicopathological characteristics and the expression of WIF-1 in gallbladder cancer.

\begin{tabular}{|c|c|c|c|c|}
\hline \multirow[b]{2}{*}{ Characteristic } & \multirow[b]{2}{*}{ Cases (n) } & \multicolumn{2}{|c|}{$\begin{array}{c}\text { Expression } \\
\text { of } W I F-1\end{array}$} & \multirow[b]{2}{*}{ P-value } \\
\hline & & - & + & \\
\hline Age (years) & & & & 0.723 \\
\hline$<60$ & 21 & 16 & 5 & \\
\hline$\geq 60$ & 29 & 24 & 5 & \\
\hline $\operatorname{Sex}$ & & & & 0.736 \\
\hline Male & 23 & 19 & 4 & \\
\hline Female & 27 & 21 & 6 & \\
\hline pT stage & & & & 0.171 \\
\hline $\mathrm{T} 1-\mathrm{T} 2$ & 20 & 14 & 6 & \\
\hline $\mathrm{T} 3-\mathrm{T} 4$ & 30 & 26 & 4 & \\
\hline Lymph node metastasis & & & & 0.171 \\
\hline Negative & 20 & 14 & 6 & \\
\hline Positive & 30 & 26 & 4 & \\
\hline Distant metastasis & & & & 0.138 \\
\hline Negative & 34 & 25 & 9 & \\
\hline Positive & 16 & 15 & 1 & \\
\hline Histological grade & & & & 0.707 \\
\hline High & 16 & 12 & 4 & \\
\hline Moderate/poor & 34 & 28 & 6 & \\
\hline
\end{tabular}

WIF-1, WNT inhibitory factor 1 .

methylation repressed the expression of WIF-1 in these cells. To further verify the presence of aberrant hypermethylation, methylation-specific PCR analysis was performed on bisulfite-modified genomic DNA harvested from the GBC cells. A high level of amplification was observed with MSPs, compared with the absence of amplification with the USPs (Fig. 1D). BSP of the NOZ promoter region was subsequently performed. As shown in Fig. 1E, the percentage of methylated $\mathrm{CpGs}$ was as high as $98.94 \%$. These findings indicated that the low expression level of WIF- 1 in the GBC cells was caused by aberrant hypermethylation of the promoter region.

Reduced expression of WIF-1 and correlation between protein levels of WIF-1 and c-Jun in GBC specimens. Negative or low expression levels of WIF-1 were observed in the cytoplasm and nucleus of the GBC mucosal cells using an immunohistochemistry technique. The expression of WIF-1 was only detected in 10 of the 50 gallbladder cancer samples, with a positive rate of $20 \%$. By contrast, positive expression was detected in the majority of the cholecystitis tissue samples $(18 / 20 ; 90 \%)$, which represented a significant difference $(\mathrm{P}<0.05)$. The associations between the expression of WIF-1 and clinicopathological characteristics of the GBC cases, including patient age and sex, $\mathrm{pT}$ stage, lymph node metastasis, distant metastasis and histological grade, are shown in Table II, which revealed that the expression of WIF-1 was not associated with any of these factors.
A high level of c-Jun-positivity was detected in serial sections of the same GBC specimens; a representative sample (case 4) is shown in Fig. 2A. By contrast, a high expression of WIF-1 and low expression of c-Jun was observed in case 26. There are currently no reports on the association between the expression of WIF-1 and c-Jun in GBC specimens. To reveal correlations between these proteins, the present study used the MOD to semiquantitatively analyze the results. The data showed a negative correlation between the protein levels of WIF-1 and c-Jun in all GBC specimens (Pearson's r $=-0.6213$; $\mathrm{P}<0.05)$, as shown in Fig. 2B.

Knockdown of c-Jun increases the $m R N A$ and protein expression of WIF-1. Among the cell lines examined (NOZ, GBC-SD and SGC-996 cells), the highest mRNA and protein expression levels of $c$-Jun, detected using RT-qPCR and western blot analyses, were found in the NOZ cells (Fig. 3A). Therefore, NOZ cells were used to further examine the role of $c$-Jun in GBC. To identify an effective siRNA for silencing the expression of $c$-Jun, three siRNA sequences (P-1, P-2 and P-3) were designed, chemically synthesized and transiently transfected into NOZ cells. The transfection efficiency was observed using fluorescence microscopy (blue light), which was high in all the groups listed above (Fig. 3B). The P-3/siRNA vector resulted in the most marked suppression of mRNA and protein expression of $c$-Jun, compared with the P-1/siRNA and P-2/siRNA vectors, whereas the NC/siRNA group had no effect (Fig. 3C). Therefore, for further experiments, P-3/siRNA was selected to induce knockdown, following which the mRNA and protein expression levels of WIF-1 were examined. As shown in Fig. 3D, the expression of WIF-1 was markedly restored in the P-3/siRNA group, which also supported the association between $c$-Jun and WIF-1 in vitro.

c-Jun represses the expression of WIF-1 by inducing hypermethylation of the WIF-1 promoter. The results of the BSP analysis of the WIF-1 promoter in P-3/siRNA-transfected and DAC-treated NOZ cells were consistent with the expression data. Hypermethylation was observed for the majority of $\mathrm{CpG}$ dinucleotides within the amplified 255 bp region of the WIF-1 promoter in NOZ cells (98.94\%; Fig. 1E), however, a low level of $\mathrm{CpG}$ methylation was observed in the $c$-Jun/siRNA (79.47\%) group $(\mathrm{P}<0.05$; Fig. 4A). Of note, the patterns of DNA methylation in the $c$-Jun/siRNA NOZ cells were most similar to those in the DAC treatment group (74.73\%; Fig. 4A), suggesting that $c$-Jun repressed the expression of WIF-1 by inducing the hypermethylation of its promoter region.

c-Jun effects the methylation of WIF-1 through transcriptional regulation and interaction with DNMT1. Methyl groups are added to CG dinucleotides by DNMT3a/3b, and DNMT1 then maintains these DNA methylation patterns during DNA replication to ensure epigenetic gene silencing (8). In the present study, RT-qPCR and western blot analyses revealed that the knockdown of $c$-Jun in NOZ cells inhibited the expression of DNMT1, but had minimal effect on the expression of DNMT3a or DNMT3b (Fig. 4B).

In addition to the regulation of DNMTs, it was hypothesized that an alternative mechanism exists, whereby $c$-Jun can physically modulate epigenetically associated proteins 
A

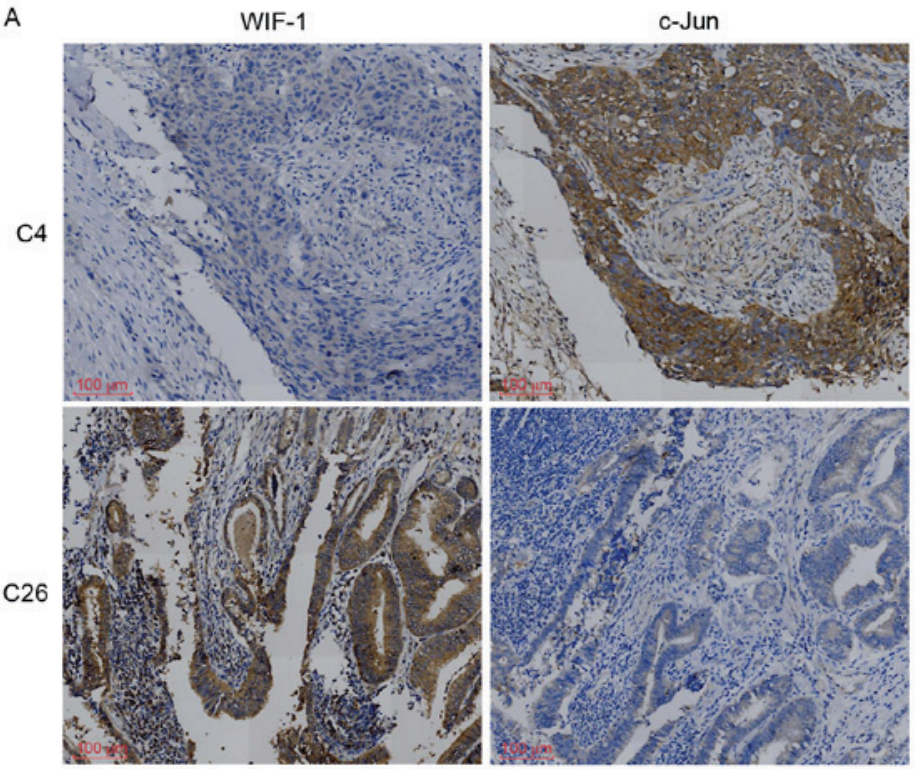

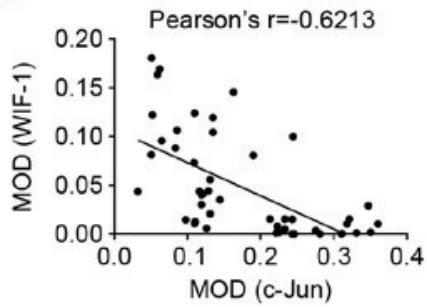

Figure 2. Correlation between protein levels of WIF-1 and c-Jun in GBC specimens. (A) Expression of WIF-1 and c-Jun were observed in the cytoplasm and nucleus of the GBC mucosa cells. C4, shows loss of expression of WIF-1 (left) with high expression of c-Jun (right). C26 shows high expression of WIF-1 (left) with low expression of c-Jun (right). (B) Protein levels of WIF-1 and c-Jun were negatively correlated in the 50 GBC specimens (Person's $r=-0.6213$; $\mathrm{P}<0.05$ ). WIF-1, WNT inhibitory factor 1; GBC, gallbladder cancer; MOD, mean optical density; C4, case 4; C26, case 26.

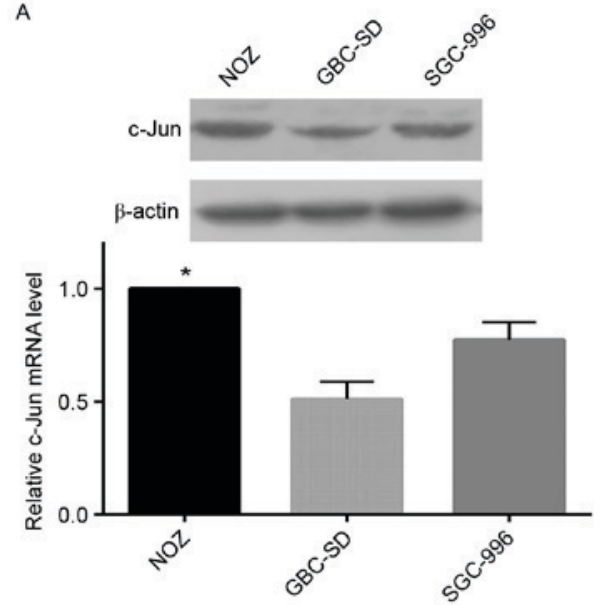

C
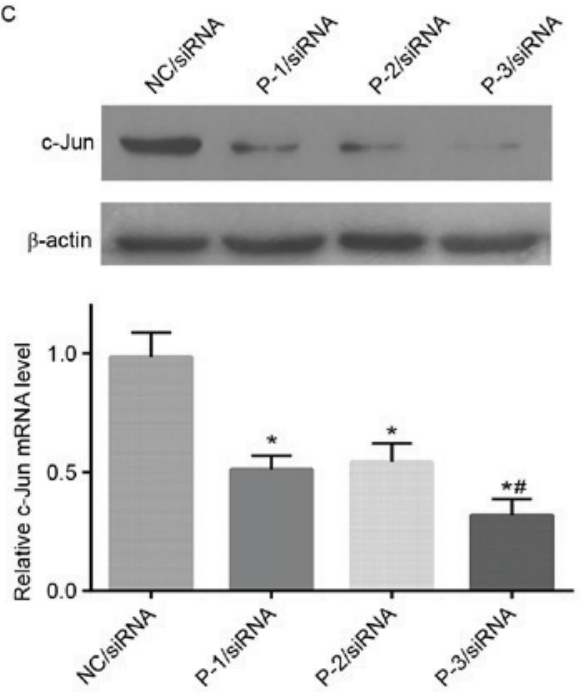

B
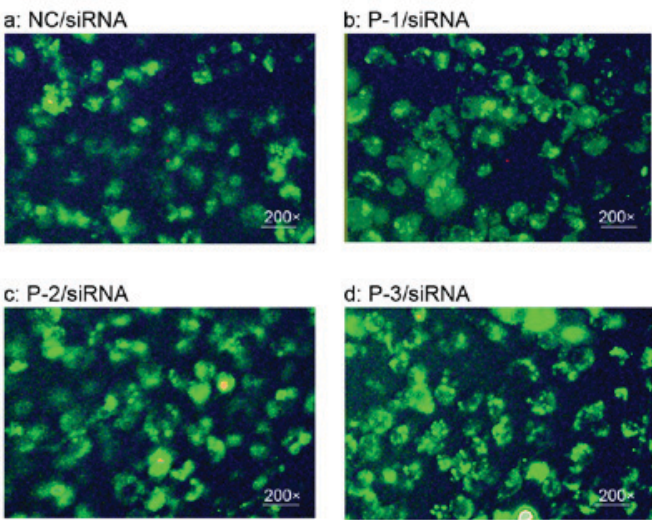

d: P-3/siRnA
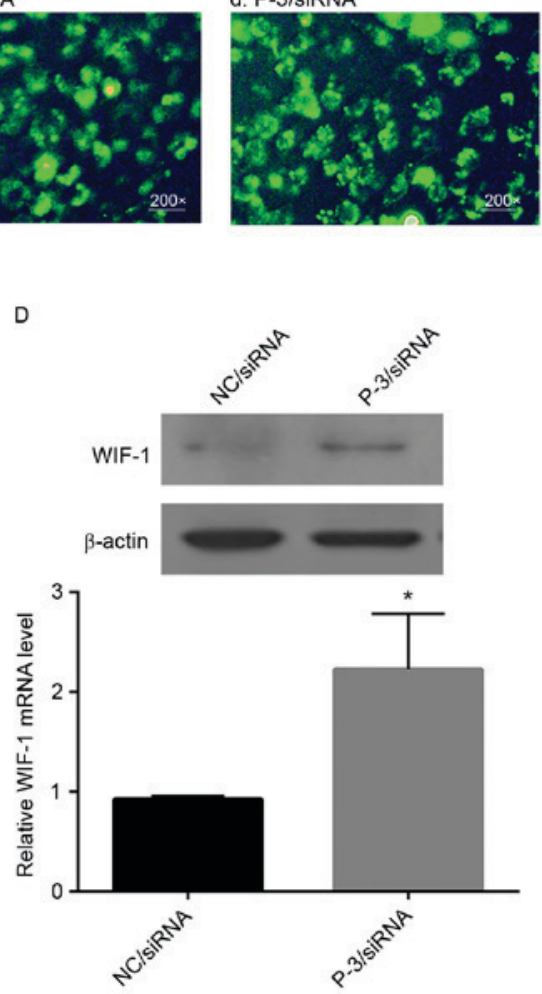

Figure 3. Knockdown of $c$-Jun affects the expression of WIF-1. (A) Relative mRNA and protein levels of $c$-Jun in GBC cell lines. "P<0.05, vs. GBC-SD and SGC-996 cells. (B) Transfection efficiency of $c$-Jun siRNA (NC/P-1/P-2/P-3) in NOZ cells. (C) Effects of $c$-Jun/siRNA transfection on the mRNA and protein expression of c-Jun. ${ }^{*} \mathrm{P}<0.05$, vs. NC/siRNA group; ${ }^{*} \mathrm{P}<0.05$, vs. $\mathrm{P}-1 /$ siRNA and $\mathrm{P}-2 /$ siRNA group. (D) Knockdown of $c$-Jun increased the mRNA and protein expression of WIF-1. ${ }^{*} \mathrm{P}<0.05$, vs. NC/siRNA group. WIF-1, WNT inhibitory factor 1; GBC, gallbladder cancer; NC, negative control; siRNA, small interfering RNA. 


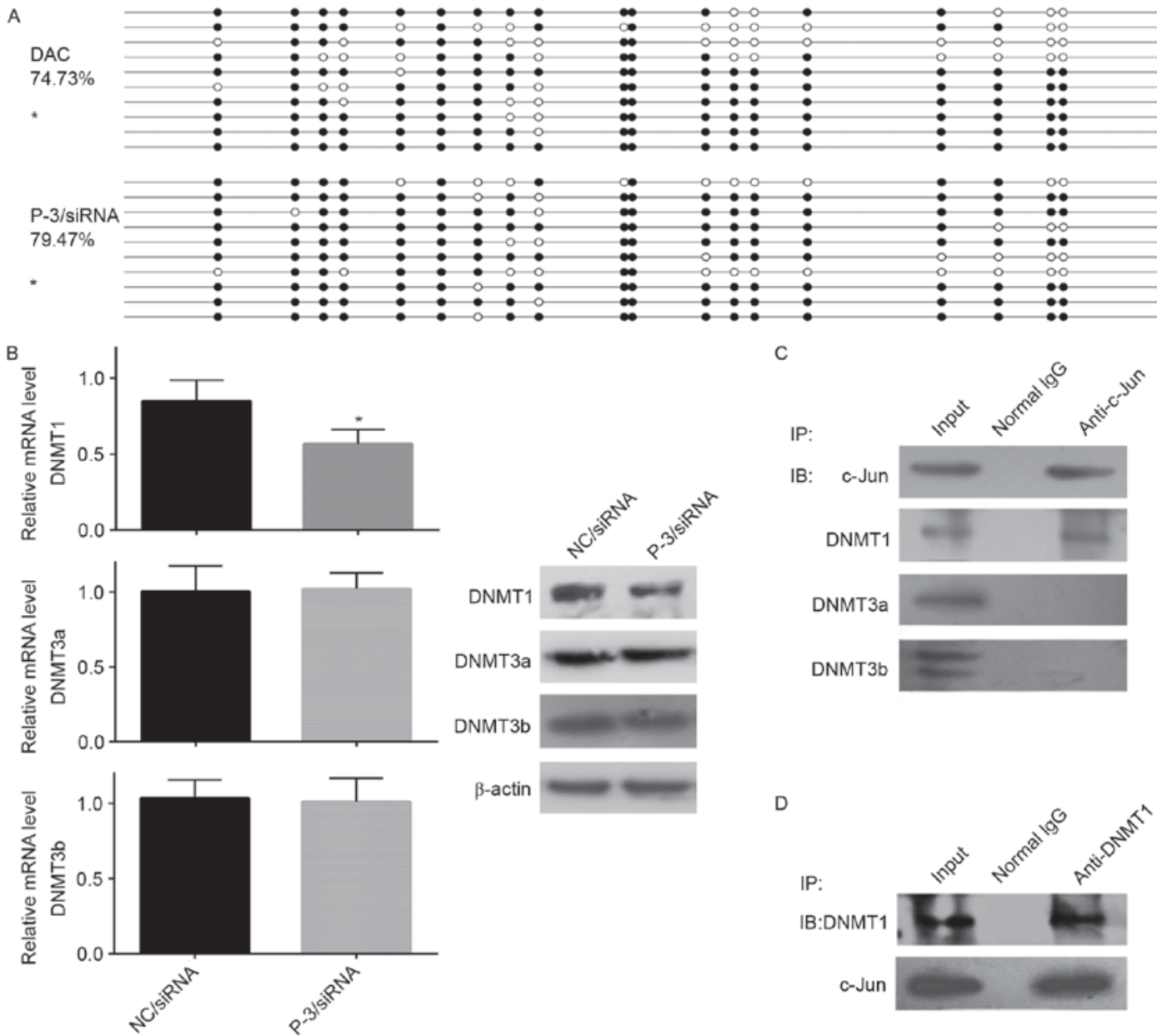

Figure 4. c-Jun affects WIF-1 methylation through transcriptional regulation and interaction with DNMT1. (A) BSP of the WIF-1 promoter region in P-3/siRNA-transfected and DAC-treated NOZ cells. Percentage of methylated Cs: DAC $=74.73 \%, \mathrm{P}-3 / \mathrm{siRNA}=79.47 \%$. ${ }^{*} \mathrm{P}<0.05$, vs. NOZ cells in Fig. $1 \mathrm{E}$. (B) Knockdown of $c$-Jun decreased the mRNA and protein expression of DNMT1, but not that of DNMT3a and DNMT3b. "P<0.05, vs. NC/siRNA group. (C) Endogenous c-Jun immunoprecipitated DNMT1, but not DNMT3a or DNMT3b. (D) Reverse immunoprecipitation was performed using anti-DNMT1 antibody. Normal IgG served as a negative control. WIF-1, WNT inhibitory factor 1; DNMT; DNA methyltransferase; GBC, gallbladder cancer; NC, negative control; siRNA, small interfering RNA.

to trigger epigenetic modifications around the regulatory elements of WIF-1. Therefore, a co-immunoprecipitation assay was designed using NOZ cells. As shown in Fig. 4C, c-Jun was immunoprecipitated with DNMT1, but not DNMT3a or DNMT3b. Reverse immunoprecipitation with DNMT1 antibodies was then performed to confirm the interaction (Fig. 4D). These results indicated that the methylation of WIF-1 associated with $c$-Jun was predominantly through DNMT1.

\section{Discussion}

WIF-1 is a key gene, which encodes a secreted protein that antagonizes the Wnt pathway and is involved in early embryonic development (25). As an important tumor suppressor, the abnormal expression of WIF-1 can result in carcinogenesis through the dysregulation of cell proliferation and differentiation. It has been demonstrated that the expression of WIF-1 is decreased in several types of tumor (10-13). The findings of the present study demonstrated a loss of the expression of WIF-1 in GBC cell lines (NOZ, GBC-SD and SGC-996). The results also showed that treatment with DAC, a potent demethylating drug, which binds to DNA methylation enzymes to inhibit their activity, restored the mRNA expression of WIF-1. In addition, the methylation-specific PCR and BSP assays showed that the hypermethylation of WIF-1 DNA may explain its loss of expression in GBC cells.

Due to the relatively low incidence, there have been few investigations of the role of WIF- 1 in the pathogenesis of GBC. In the present study, the protein expression of WIF-1 was examined in $50 \mathrm{GBC}$ and 20 cholecystitis specimens using imunohistochemical techniques. A reduced expression of WIF-1 was detected in the GBC specimens, which indicated that WIF-1 was important in the pathogenesis of GBC. However, in contrast to previous reports of other tumors, subgroup analysis showed no correlation between the expression of WIF-1 and key clinicopathological parameters in GBC, including age and sex, pT stage, lymph node metastasis, distant metastasis and histological grade. This suggested that WIF-1 may be involved in the tumorigenesis of GBC only, and not the progression.

There have been few investigations to date regarding the upstream molecular mechanism for regulating WIF-1. In the present study, it was observed that the protein levels of WIF-1 and c-Jun were negatively correlated in serial 
sections of 50 GBC specimens, suggesting an association between $c$-Jun and WIF-1. RNA interference was used to for the in vitro knockdown $c$-Jun, and an effective siRNA was obtained (P-3/siRNA). The mRNA and protein expression levels of $W I F-1$ were then detected in NOZ cells transiently transfected with $\mathrm{P}-3 /$ siRNA, and the results demonstrated that silencing of the $c$-Jun gene upregulated the expression of $W I F-1$. Considering this evidence, it was hypothesized that $c$-Jun may be involved in regulating the expression of WIF-I in GBC.

The results described above prompted further examination of the specific molecular mechanism underlying the $c$-Jun-induced downregulated expression of WIF-1. The epigenetic modification of cellular genes via aberrant methylation in promoter regions has been identified as a crucial mechanism for inactivating tumor suppressor genes in tumors $(4,5,26)$. The present study showed silencing of WIF-I through hypermethylation of its promoter region in GBC cells. With these previous findings in consideration, it was hypothesized that $c$-Jun repressed the expression of WIF-1 by modifying the promoter methylation status of the WIF-1 gene. To confirm the factors involved, BSP assays were performed, and the obtained data showed that $\mathrm{CpG}$ islands in the WIF-1 promoter region were hypermethylated at a higher frequency in NOZ cells, compared with those in the knockdown group (P-3/siRNA cells), revealing a similar methylation ratio to that observed in the DAC-treated group. These results suggested that the knockdown of $c$-Jun caused the demethylation of $\mathrm{CpG}$ islands in the $W I F-1$ promoter region. Taken together, these findings are confirmed the hypothesis that $c$-Jun can repress the expression of WIF-1 through epigenetic modification by inducing hypermethylation of the WIF-1 promoter in GBC.

Methylation modifications are mediated by DNMTs through various mechanisms. DNMT1 and DNMT3b have been shown to be responsible for the aberrant hypermethylation of WIF-I in the HCT116 colon cancer cell line (10). The present study investigated whether $c$-Jun affected DNMTs in GBC cells The experimental results demonstrated that $c$-Jun knockdown in the NOZ cells inhibited the expression of DNMT1, but not that of DNMT3a or DNMT3b. A co-immunoprecipitation assay was performed to examine the mechanism involved in the chromatin remodeling induced by $c$-Jun, and it was found that c-Jun interacted with DNMT1 only, and not with DNMT3a or DNMT3b. Taken together, these results showed that $c$-Jun affects the methylation of WIF-1 through transcriptional regulation and interaction with DNMT1, a critical DNA methyltransferase.

However, the results of the present study were based on data obtained using GBC cells and partial GBC tissues. Unlike the findings in the present study, a previous study reported that $W I F-1$ was expressed in the majority of colorectal cancer specimens (27). The downregulation of $c$-Jun has been observed in specific types of human cancer, including breast cancer; these discrepancies may be due to the different types and developmental stages of the cells examined $(28,29)$. Therefore, the regulatory mechanisms of $c$-Jun and WIF-1 are complicated, however, the results of the present study assist in elucidating the mechanisms linking these proteins in GBC.

In conclusion, the present study found that the expression of WIF-1 was low in GBC cells due to aberrant hypermethylation of its promoter region. Additionally, an alternative pathogenesis of GBC was indicated in which $c$-Jun causes hypermethylation of the WIF-1 promoter region, and represses the expression of WIF-1 through transcriptional regulation and interaction with DNMT1 as an early event in the tumorigenesis of GBC.

\section{Acknowledgements}

The present study was supported by grants from the National Natural Science Foundation of China (grant no. 81672468) and the National Clinical Key Specialty Construction Project (General Surgery) of China (grant no. 2012-649). This manuscript was edited for English language by American Journal Experts (Durham, NC, USA).

\section{References}

1. Lazcano-Ponce EC, Miquel JF, Muñoz N, Herrero R, Ferrecio C, Wistuba II, Alonso de Ruiz P, Aristi Urista G and Nervi F: Epidemiology and molecular pathology of gallbladder cancer. CA Cancer J Clin 51: 349-364, 2001.

2. Sheth S, Bedford A and Chopra S: Primary gallbladder cancer: Recognition of risk factors and the role of prophylactic cholecystectomy. Am J Gastroenterol 95: 1402-1410, 2000.

3. Levy AD, Murakata LA and Rohrmann CA Jr: Gallbladder carcinoma: Radiologic-pathologic correlation. Radiographics 21: 295-314, 2001.

4. Schroeder $\mathrm{M}$ and Mass $\mathrm{MJ}$ : $\mathrm{CpG}$ methylation inactivates the transcriptional activity of the promoter of the human p53 tumor suppressor gene. Biochem Biophys Res Commun 235: 403-406, 1997.

5. Rizvi MM, Alam MS, Ali A, Mehdi SJ, Batra S and Mandal AK: Aberrant promoter methylation and inactivation of PTEN gene in cervical carcinoma from Indian population. J Cancer Res Clin Oncol 137: 1255-1262, 2011.

6. Oshimo Y, Nakayama H, Ito R, Kitadai Y, Yoshida K, Chayama K and Yasui W: Promoter methylation of cyclin D2 gene in gastric carcinoma. Int J Oncol 23: 1663-1670, 2003.

7. Mazieres J, He B, You L, Xu Z, Lee AY, Mikami I, Reguart N, Rosell R, McCormick F and Jablons DM: Wnt inhibitory factor-1 is silenced by promoter hypermethylation in human lung cancer. Cancer Res 64: 4717-4720, 2004.

8. Chen T and Li E: Structure and function of eukaryotic DNA methyltransferases. Curr Top Dev Biol 60: 55-89, 2004.

9. Kawano Y and Kypta R: Secreted antagonists of the Wnt signalling pathway. J Cell Sci 116: 2627-2634, 2003.

10. Ai L, Tao Q, Zhong S, Fields CR, Kim WJ, Lee MW, Cui Y, Brown KD and Robertson KD: Inactivation of Wnt inhibitory factor-1 (WIF1) expression by epigenetic silencing is a common event in breast cancer. Carcinogenesis 27: 1341-1348, 2006.

11. Mazieres J, He B, You L, Xu Z, Lee AY, Mikami I, Reguart N, Rosell R, McCormick F and Jablons DM: Wnt inhibitory factor-1 is silenced by promoter hypermethylation in human lung cancer. Cancer Res 64: 4717-4720, 2004.

12. Ramachandran I, Thavathiru E, Ramalingam S, Natarajan G, Mills WK, Benbrook DM, Zuna R, Lightfoot S, Reis A, Anant S and Queimado L: Wnt inhibitory factor 1 induces apoptosis and inhibits cervical cancer growth, invasion and angiogenesis in vivo. Oncogene 31: 2725-2737, 2012.

13. Huang Y, Du Q, Wu W, She F and Chen Y: Rescued expression of WIF-1 in gallbladder cancer inhibits tumor growth and induces tumor cell apoptosis with altered expression of proteins. Mol Med Rep 14: 2573-2581, 2016.

14. Dérijard B, Hibi M, Wu IH, Barrett T, Su B, Deng T, Karin M and Davis RJ: JNK1: A protein kinase stimulated by UV light and Ha-Ras that binds and phosphorylates the c-Jun activation domain. Cell 76: 1025-1037, 1994.

15. Behrens A, Sibilia M and Wagner EF: Amino-terminal phosphorylation of c-Jun regulates stress-induced apoptosis and cellular proliferation. Nat Genet 21: 326-329, 1999.

16. Behrens A, Jochum W, Sibilia M and Wagner EF: Oncogenic transformation by ras and fos is mediated by c-Jun N-terminal phosphorylation. Oncogene 19: 2657-2663, 2000. 
17. Schreiber M, Kolbus A, Piu F, Szabowski A, Möhle-Steinlein U, Tian J, Karin M, Angel P and Wagner EF: Control of cell cycle progression by c-Jun is p53 dependent. Genes Dev 13: 607-619, 1999.

18. Ryan KM, Phillips AC and Vousden KH: Regulation and function of the p53 tumor suppressor protein. Curr Opin Cell Biol 13: 332-337, 2001.

19. Davis RJ: Signal transduction by the JNK group of MAP kinases. Cell 103: 239-252, 2000.

20. Vogt PK: Jun, the oncoprotein. Oncogene 20: 2365-2377, 2001.

21. Livak KJ and Schmittgen TD: Analysis of relative gene expression data using real-time quantitative PCR and the 2(-Delta Delta C(T)) method. Methods 25: 402-408, 2001.

22. Rice P, Longden I and Bleasby A: EMBOSS: The european molecular biology open software suite. Trends Genet 16: 276-277, 2000.

23. Li LC and Dahiya R: MethPrimer: Designing primers for methylation PCRs. Bioinformatics 18: 1427-1431, 2002.

24. BockC, Reither S, Mikeska T, Paulsen M, Walter J and Lengauer T: BiQ Analyzer: Visualization and quality control for DNA methylation data from bisulfite sequencing. Bioinformatics 21: 4067-4068, 2005.
25. Hsieh JC, Kodjabachian L, Rebbert ML, Rattner A, Smallwood PM, Samos CH, Nusse R, Dawid IB and Nathans J: A new secreted protein that binds to Wnt proteins and inhibits their activities. Nature 398: 431-436, 1999.

26. Li HP, Leu YW and Chang YS: Epigenetic changes in virus-associated human cancers. Cell Res 15: 262-271, 2005.

27. Byun T, Karimi M, Marsh JL, Milovanovic T, Lin F and Holcombe RF: Expression of secreted Wnt antagonists in gastrointestinal tissues: Potential role in stem cell homeostasis. J Clin Pathol 58: 515-519, 2005.

28. Smith LM, Birrer MJ, Stampfer MR and Brown PH: Breast cancer cells have lower activating protein 1 transcription factor activity than normal mammary epithelial cells. Cancer Res 57: 3046-3054, 1997.

29. Kharman-Biz A, Gao H, Ghiasvand R, Zhao C, Zendehdel K and Dahlman-Wright K: Expression of activator protein-1 (AP-1) family members in breast cancer. BMC cancer 13: 441, 2013. International (CC BY-NC-ND 4.0) License. 